\title{
EDITORIALE
}

\section{NEURO SHOPPING: COME E PERCHÉ ACQUISTIAMO}

\author{
Gianpiero Lugli*
}

Per comprendere lo scostamento tra le risposte che il consumatore dà verbalmente in occasione di interviste - focus group e il suo comportamento reale in punto vendita, occorre rivisitare il marketing distributivo attraverso la lente della psicologia cognitiva, della economia comportamentale e delle neuroscienze.

I distributori hanno sempre orientato le loro azioni di marketing in funzione del comportamento a-razionale del consumatore. Molto prima della nascita della psicologia cognitiva, delle neuroscienze e dell'economia comportamentale, i distributori costruivano infatti le loro proposte di valore tenendo conto delle componenti emotive dell'acquisto e della possibilità di condizionare la mente cognitiva per attrarre, fidelizzare e indurre il consumatore ad acquistare più del necessario. In un mercato competitivo, le insegne sono obbligate a prestare grande attenzione al comportamento del consumatore sperimentando continuamente nuove soluzioni per battere $\mathrm{i}$ rivali nella conquista del gradimento della clientela oltre che nel condizionamento del comportamento di acquisto. La sperimentazione e l'apprendimento, che discende dall'analisi dell'efficacia delle azioni intraprese, hanno finito per orientare le insegne verso politiche di marketing che sembrano ispirate dalla conoscenza del funzionamento della mente del consumatore quando decide dove, cosa e quanto acquistare. Si pensi per esempio alla pratica di utilizzare sequenze terminali composte da decimali invece che da numeri interi. L'esperienza nella vendita ha insegnato alle insegne che la tecnica del digit o 9-ending pricing induce il consumatore a percepire un minor esborso; le imprese sono state cioè in grado di assumere comportamenti efficaci anche senza disporre di prove empiriche per validare

* Professore ordinario, Dipartimento di Economia, Università degli Studi di Parma, email: gianpiero.lugli@unipr.it.

Mercati e Competitività n. 4, 2010 


\section{G. Lugli}

queste rules of thumbs ${ }^{1}$. Un altro caso emblematico è offerto dalla vecchia tecnica del prendi tre e paghi due. Si è sempre detto che con questa tecnica promozionale il distributore ed il fornitore che contribuisce al finanziamento vogliono:

- stimolare la penetrazione attirando i non acquirenti;

- stoccare gli acquirenti abituali sottraendoli di conseguenza ai competitor almeno fino all'esaurimento della scorta di consumo.

Ovviamente, negli affari non esistono regali. Eppure, molte proposte promozionali contengono la parola magica "costo zero" o "gratis". Lo zero ha un significato matematico, ma non può esprimere il valore di un oggetto che ha una qualche utilità per chi lo acquista. Le offerte del tipo "paghi 2 e prendi 3 " vorrebbero farci percepire che il terzo prodotto non costa nulla. In realtà, anche il terzo prodotto ha un costo in quanto per ottenerlo dobbiamo ottemperare ad una condizione onerosa sia in termini monetari che di vincolo al consumo futuro, visto che dovremo smaltire la scorta prima di acquistare una marca alternativa. Ciò non di meno, il regalo ci colpisce emotivamente con un'eccitazione del tutto irrazionale. La promozione multibuy, così come l'offerta di confezioni con una grammatura extra in omaggio, è una tecnica promozionale che non viene impiegata da tutte le marche, ma solo da quelle con una bassa penetrazione e un'altrettanto bassa quota di mercato. Ciò in quanto, facendo leva sulla nostra prevedibile "irrazionalità", il venditore riesce a farci assumere comportamenti contrari ai nostri reali giudizi sulle alternative offerte. In altri termini, se invece di offrire 3 prodotti al prezzo di due, il distributore avesse semplicemente ridotto di un terzo il prezzo di vendita, avremmo continuato ad acquistare la marca leader. Siccome un prodotto ci viene presentato con la parolina magica (gratis), ecco che la nostra mente emotiva si eccita al punto che ci fa acquistare un prodotto che, se fosse stato proposto con un prezzo trasparente, non avremmo mai acquistato.

Del tutto analoghe sono le offerte di pagamento rateale a tasso zero. In questo caso siamo addirittura in presenza di un insulto alla mente cognitiva, visto che non basta evitare di esporre il tasso di interesse per poter affermare di averlo azzerato. Il principio è comunque lo stesso. Si offre la possibilità di godere oggi e rinviare il sacrificio nel futuro e, vista l'asimmetria con cui funziona il nostro cervello emotivo nel valutare il presente rispetto al futuro, molti si fanno abbindolare.

Il distributore ha scoperto dunque, attraverso un meccanismo di continua sperimentazione e correzione degli errori, come funziona la mente del consumatore quando decide dove, cosa e quanto acquistare, ma le opportu-

1. Bitzer G.Y., Schindler R.M. (2005), Direct evidence of ending-digit drop-off in price information processing, Psycol Mark 22: 771-802. 
nità di ulteriore miglioramento sono consistenti. È compito del marketing aiutare le imprese e gli studenti a comprendere le radici psicologiche e neurobiologiche del comportamento di acquisto. La complessità del comportamento di acquisto e di consumo sono tali che, se vogliamo migliorare la nostra comprensione del mercato, non possiamo adottare un solo punto di vista. Occorre invece porsi da più prospettive, unire i saperi, le metodologie, fondere ciò che ognuna di esse riesce a cogliere, in modo necessariamente parziale ma altrettanto necessariamente complementare. La psicologia e le neuroscienze hanno aperto nuove prospettive per l'economia e il marketing, dal momento che hanno dimostrato che:

- la decisione economica scaturisce dalla combinazione di processi controllati e automatici, cognitivi ed emotivi;

- il processo decisionale ha una importante componente relazionale per la nostra capacità di rappresentare la mente altrui;

- il contesto con cui si presenta una opzione condiziona la decisione e mette dunque in discussione l'assioma economico della invarianza.

In particolare, spetta al marketing distributivo offrire gli strumenti logici per comprendere la perdita di valore che le insegne subiscono quando rinunciano alla loro autonomia per cogliere le opportunità offerte dall'industria sotto forma di incentivi e sconti legati a prestazioni. Il trade marketing industriale rappresenta infatti un potente ostacolo al pieno orientamento del marketing distributivo verso la mente dell'acquirente. Certo, le insegne accettano il condizionamento del trade marketing in diversa misura a seconda del gruppo strategico di appartenenza e della loro cultura aziendale. Tutte le insegne sono però sensibili alle lusinghe dei contributi marketing dell'industria ${ }^{2}$, proprio perché utilizzando i paradigmi tradizionali non è possibile valutare i costi della rinuncia all'autonomia del marketing distributivo. Se non si estende il contenuto del marketing distributivo alle conoscenze prodotte dalle discipline che si occupano del funzionamento della mente, non si riesce a comprendere la perdita di valore che le insegne subiscono accettando il condizionamento industriale. Il caso più emblematico, perché riguarda tutte le insegne, è l'eccessiva estensione in ampiezza, varietà e profondità, dell'assortimento di categoria. I contributi offerti dall'industria per inserire marche poco richieste, ovvero per estendere la copertura in termini di segmenti di consumo e formati di una marca già trattata, dovrebbero essere confrontati con gli oneri generati dall'eccesso di scelta. L'idea secondo cui quanto è maggiore la scelta, tanto più ampia è la soddisfazione potenziale del consumatore, riposa sull'ipotesi di razionalità della decisione; infatti, se prima di decidere analizzassimo tutte le alternative

2. L'unica eccezione è rappresentata dalle insegne che operano nel formato dell'hard discount. 


\section{G. Lugli}

sulla base delle informazioni disponibili e di processi logici, aumentando il numero delle alternative cresce anche la probabilità che nell'insieme compaia il prodotto in grado di soddisfare maggiormente le aspettative dell'acquirente. Purtroppo, o per fortuna, le cose non stanno così. Non è vero che aumentando ampiezza, profondità e varietà dell'assortimento di categoria migliora di conseguenza la soddisfazione del consumatore. La nostra mente cognitiva ha infatti limiti di capacità elaborativa e vincoli di tempo; la scelta è pertanto realizzata in modo a-razionale. La continua crescita degli assortimenti commerciali non può essere tuttavia ricondotta solo alla mancata comprensione del processo di scelta del consumatore e, quindi, degli effetti negativi che l'eccesso di alternative può avere sulle vendite. Spesso si tratta infatti di una scelta ponderata tra due benefici alternativi entrambi ricercati dall' acquirente: il prodotto e la convenienza di prezzo. Questo trade off contiene però un bias cognitivo dal momento che è facile quantificare i premi di referenziamento dell'industria, ma non le conseguenze di un eccesso di scelta. Solo l'analisi psicologica e neurologica può contribuire a quantificare l'ossimoro del more is less. Alcuni esperimenti psicologici hanno dimostrato che l'eccesso di scelta induce il consumatore a rinunciare all'acquisto della categoria ${ }^{3}$. Siccome esiste una sostituibilità delle categorie nella funzione d'uso e, soprattutto, siccome il compito del marketing distributivo dovrebbe essere innanzitutto quello di indurre il consumatore a superare l'opzione zero del non acquisto, si può ben capire che la perdita di valore generata da un eccesso di scelta può essere notevole. Altre volte, l'eccesso di scelta obbliga ad adottare euristiche a-razionali per effettuare una scelta compatibile coi vincoli di tempo. Una pizzeria di via D'Azeglio a Parma offre stabilmente un menu di 52 alternative a consumatori che non hanno il tempo, e la voglia, di analizzare tutte le proposte per fare la propria scelta. Anche questo è un caso in cui more is less. Invece di catturare l'attenzione del consumatore segmentando l'offerta in categorie di acquisto o variabilizzando temporalmente le proposte offrendo solo alcune alternative al giorno, la pizzeria di via D'Azeglio offre tutte le pizze contemporaneamente e indistintamente ad un consumatore frettoloso che finisce per trasformare la ricchezza della proposta commerciale in povertà relazionale.

Un'altra area del marketing distributivo in cui si può constatare la perdita di valore per effetto delle interferenze industriali è quella della promozione. I contributi offerti dai produttori per scontare il prezzo dei loro prodotti sono ovviamente giustificati dal punto di vista industriale, ma non è

3. Iyengar S.S. and Lepper M.R. When choice is demotivating: can one desire too much of a good thing?, Journal of Personality and Social Psychology, 2000, Volume 79, number 6, pp. 995-1006. Schwartz B. (2004), The paradox of choice: why more is less, Ecco, Ney York. Miller G.A. (1956). The magic number seven plus or minus two: some limits in our capacity for processing information. Psychol Rev 63: 81-97. Gigerenzer G. (2009). Decisioni Intuitive, Raffaello Cortina Editore, p. 32 
detto che siano convenienti anche dal punto di vista commerciale. Le insegne non "vendono" infatti singoli prodotti, ma assortimenti di categoria e di punto vendita. In altre parole, lo sconto di un prodotto che il consumatore non è interessato ad acquistare genera un modesto impatto sul traffico, sulla fidelizzazione e sullo scontrino. Secondo un'indagine commissionata da Carrefour all'IFOP all'inizio del 2010, il 41\% dei 1155 consumatori intervistati ritiene che le promozioni offerte non corrispondano alle loro reali necessità ovvero riguardino prodotti non acquistati abitualmente ${ }^{4}$. Forte dei risultati di questo sondaggio, Carrefour ha deciso di reinventare la promozione offrendo al consumatore la possibilità di scegliere direttamente $\mathrm{i}$ prodotti da acquistare in sconto all'interno di reparti (macrocategorie) definiti settimanalmente. Il nuovo concept (PromoLibre) è stato lanciato nella settimana dal 23 al 28 febbraio 2010 sulla prima colazione (650 referenze) e sui piccoli elettrodomestici. Nelle macrocategorie del grocery, il consumatore può liberamente scegliere tre diversi prodotti e Carrefour rimborserà il meno costoso con un accredito di punti sulla fidelity card. Nelle macrocategorie non-grocery che settimanalmente entrano nell'iniziativa PromoLibre, il consumatore può acquistare singoli prodotti con lo sconto del $20 \%$ che gli viene accreditato in punti sulla fidelity card. Non sappiamo se Carrefour abbia realizzato un accordo coi fornitori dei prodotti selezionati in promo dal consumatore per ottenere un contributo ex post; non ci stupirebbe però la presenza di un tale accordo. Ciò che ci preme sottolineare di questa innovazione di marketing è il cambiamento di prospettiva:

- invece di incentivare i consumatori ad acquistare prodotti che altrimenti non avrebbero acquistato, si offre convenienza partendo dalle reali preferenze del consumatore e sfruttando il principio psicologico del debito;

- invece di promuovere la vendita di singoli prodotti, si offre convenienza e di conseguenza si sostengono le vendite di intere categorie con una frequenza settimanale, in modo da mantenere nel tempo l'interesse del consumatore per effettuare la spesa presso l'insegna;

- invece di stimolare la penetrazione e l'up selling con l'offerta di tre pezzi di una stessa referenza al prezzo di due, si offre al consumatore la possibilità di costruire il paniere di prodotti che preferisce all'interno di un ampio campo di scelta;

- invece di stimolare la vendita di predefiniti prodotti non food offrendo semplicemente uno sconto di prezzo, si offre al consumatore la possibilità di scegliere il prodotto su cui redimere lo sconto creando così un forte incentivo al superamento dell'opzione zero, che è rappresentata dal non acquisto; calcolando poi lo sconto in moneta commerciale espressa dai punti carta, l'insegna può governare la redemption dello sconto in funzione dei suoi obiettivi di marketing.

4. Colloquy, 02/23/2010. 


\section{G. Lugli}

Qualche ulteriore precisazione è necessaria per chiarire il meccanismo psicologico del debito. Dal momento che il consumatore può decidere cosa acquistare in sconto, la promozione non viene percepita come una transazione in cui l'insegna offre un vantaggio a chi compra un prodotto che non avrebbe altrimenti acquistato. Con questo orientamento prosumerista, la promozione viene percepita come un regalo dell'insegna che, in questo modo, attiva l'obbligazione di reciprocità trasformando una relazione commerciale in una relazione personale attraverso una manifestazione di interesse e simpatia. Molto più diffusa è la tecnica del campione omaggio offerto dal produttore o dal distributore, ufficialmente per far conoscere, ma in realtà per aumentare la penetrazione del prodotto; anche in questo caso, il regalo attiva una obbligazione di reciprocità.

Occorre poi conoscere la mente del cliente anche per individuare i lavori che può svolgere volentieri, sostituendo così il personale di vendita. Oltre alla necessità di conoscere la mente del cliente per comprendere (e influenzare) il comportamento di acquisto, vi è la necessità di tener conto delle componenti psicologiche e relazionali per decidere il suo coinvolgimento diretto nella produzione di valore economico. Il fatto che il consumatore non si limiti a consumare ma contribuisca alla produzione assumendo il ruolo di prosumer e, analogamente, il fatto che il consumatore non si limiti a comprare quando si trova nel punto vendita ma sostituisca il personale dipendente in alcuni aspetti della produzione del servizio ed assuma quindi il ruolo di working shopper, non deve trarre in inganno. Il coinvolgimento del consumatore nella configurazione dell'output non solo deve incontrare la sua disponibilità, ma deve essere realizzato con modalità che il cliente percepisce come una soluzione obbligata per migliorare la sua soddisfazione nel servizio e/o nel prezzo. Il consumatore che usa il mobile self scanning sostituendo così il personale dipendente nella funzione di price look up, ha un ritorno nel minor tempo che impiega per fare la spesa. Lo stesso si può dire per il consumatore che, invece di acquistare l'ortofrutta a libero servizio, sceglie direttamente i prodotti, che poi pesa e prezza, per governare meglio la quantità e la qualità. Il consumatore IKEA che trasporta il mobile smontato fino al suo domicilio dove lo rimonta, oltre a riportarlo in punto vendita nel caso vengano riscontrati difetti, ottiene come corrispettivo un minor prezzo rispetto alle altre alternative. Occorre dunque conoscere la mente del cliente per individuare i lavori che può svolgere volentieri, le modalità ed il ritorno del coinvolgimento. Lo sviluppo di opportunità di working shopper che finiscono per coinvolgere una quota significativa della clientela rappresenta un'innovazione transazionale di grande importanza per la realizzazione di un vantaggio competitivo sostenibile. In un'economia di mercato che funziona bene, non esiste il rischio di uno "sfruttamento" del consumatore che lavora nella produzione di beni e servizi; esistono, infatti, sempre di- 
versi livelli di prosumerismo tra cui il consumatore può scegliere semplicemente cambiando insegna. Di nuovo, per governare con successo la leva del coinvolgimento del consumatore nella produzione del servizio commerciale, occorre conoscere le basi psicologiche e neurologiche della percezione di valore.

È giunto il momento di esplorare la possibilità di un nuovo approccio alla segmentazione di marketing, basato sul processo mentale che porta all'acquisto piuttosto che sul comportamento ex post dell' acquirente. Dalla comprensione del funzionamento della mente del consumatore quando deve scegliere tra diverse alternative, discende la possibilità di individuare la differenziazione delle persone in materia di comportamento di acquisto, aggregandole poi in segmenti utili per orientare l'attività di marketing. Inoltre, dalla comprensione delle euristiche psicologiche del comportamento umano, è possibile prevedere l'efficacia degli stimoli economici. In realtà, più che di una messa in discussione del principio economico della razionalità e della funzione di utilità come fondamento del processo decisionale, si può parlare di un'estensione di questi concetti per/al fine di migliorare la loro capacità di prevedere i comportamenti umani nella sfera economica. L'importanza dei fattori emotivi nell'orientare il comportamento di acquisto dev'essere poi combinata col fatto che gli individui non decidono in isolamento, ma in presenza di altri e, quindi, il loro comportamento si basa anche su un processo di interazione. La nostra capacità di rappresentare gli stati mentali altrui sta alla base delle relazioni cooperative e conflittuali, ma non mette in discussione la funzione di utilità attesa su cui si basano le decisioni economiche; semplicemente, occorre aggiungere un ulteriore argomento/tassello (relazionale) alla funzione che governa le nostre scelte economiche. Nel caso dell'acquisto di un bene, l'attivazione delle aree cerebrali che prevedono il piacere del consumo (nucleus accumbens) dev'essere comparata con l'attivazione delle aree che anticipano il dolore del prezzo da pagare (insula). Questa controversia neuronale della mente emotiva è spesso sufficiente per decidere l'acquisto, ma a volte è accompagnata dall'intervento della mente consapevole (corteccia prefrontale) se si tratta di acquistare un prodotto di cui non abbiamo esperienza di consumo e/o di valutare la convenienza del prezzo rispetto ad offerte spaziotemporali alternative ${ }^{5}$.

Nella letteratura di marketing distributivo, la segmentazione degli acquirenti viene tradizionalmente effettuata in base:

- ai benefici ricercati a livello di singolo prodotto attraverso l'analisi della composizione della spesa (Tesco);

5. Knutson B., Rick S., Wimmer G.E. et al. (2007). Neural Predictors of Purchases. Neuron, 53: 147-156. 
- all'importanza per l'insegna in termini di frequenza e recenza, scontrino e spesa nell'unità di tempo, contribuzione media;

- allo stile espresso dalla sensibilità al prezzo e al branding distributivo.

Il Neuroshopping si propone fra l'altro di dimostrare l'utilità di un nuovo approccio alla segmentazione della clientela, che prescinde dall'analisi del comportamento effettivo ricavabile dai data base e si focalizza per contro sull'utilizzo delle diverse componenti della mente e sulle euristiche psicologiche nel processo di acquisto. Questa nuova segmentazione della clientela non sostituisce la segmentazione tradizionale, ma si aggiunge a questa e può portare notevoli benefici nella realizzazione dell'obiettivo dell'up selling. Posto che gran parte degli acquisti è decisa all'interno del punto vendita, occorre una segmentazione della clientela che tenga conto delle dimensioni psicologiche, neurologiche e relazionali, per spingere il consumatore ad aumentare i suoi acquisti nel momento in cui entra in contatto con l'offerta commerciale.

A tutti noi piace pensare di essere razionali. In fondo, ciò che distingue l'uomo dagli animali è proprio la capacità riflessiva ed il ricorso alla logica. Si è dunque consolidata l'idea che la bontà di una decisione è direttamente legata alla razionalità del processo logico che sta alla sua base. $\mathrm{Ne}$ consegue che le emozioni sono sempre state considerate, fin dai tempi di Platone, come un elemento di disturbo all'assunzione di comportamenti razionali e virtuosi. I filosofi e gli psicologi tendono a rafforzare la separazione platonica tra ragione ed emozioni privilegiando la prima, in quanto sono convinti che i sentimenti interferiscano con la mente consapevole e debbano, di conseguenza, essere tenuti "imbrigliati" per adottare scelte razionali.

La comprensione del comportamento umano in tutta la sua complessità richiede di superare la tradizionale dicotomia razionalità-irrazionalità; esiste infatti una dimensione a-razionale nelle nostre decisioni di acquisto, che è condizionata da determinanti psicologiche ed emotive. Lo stesso Nietzche sosteneva che "L'irrazionalità di una cosa non è argomento contro la sua esistenza anzi ne è la condizione." Il fatto poi che molte decisioni siano assunte a-razionalmente non vuol dire che siano sbagliate, anzi, si può dimostrare che il solo approccio razionale non permette in alcuni casi di effettuare la scelta giusta nei tempi richiesti ${ }^{6}$.

La psicologia cognitiva e le neuroscienze hanno aperto nuove prospettive per l'economia e il marketing, dal momento che numerosi esperimenti hanno dimostrato che il contesto (frame) e la rappresentazione delle opzioni condizionano la decisione, mettendo dunque in discussione l'assioma economico dell'invarianza. Le conoscenze prodotte dalla psicologia cogni-

6. Lehrer J. (2009), Come decidiamo, Codice Edizioni (op. cit.). 
tiva e dalle neuroscienze hanno permesso lo sviluppo di una nuova branca dell'economia: l'economia comportamentale (Behavioural economics). Diversamente dall'economia tradizionale, che non si occupa del processo con cui l'individuo arriva alla scelta perché si limita ad analizzare le preferenze rivelate ed il loro legame con l'utilità attesa, il nuovo approccio si propone di comprendere i meccanismi che presiedono alla scelta. Nel marketing, che può essere considerato come un segmento operativo dell'economia comportamentale, questa comprensione è fondamentale per orientare il comportamento dell'impresa in rapporto a preferenze instabili e condizionabili. Secondo la teoria delle preferenze rivelate, infatti, se un consumatore sceglie il prodotto $\mathrm{x}$, si può derivare che lo preferisca agli altri sostituti e questa preferenza resti stabile nel tempo. Al contrario, nella realtà si osserva invece che il consumatore si sposta facilmente da una marca all'altra; questo non significa che le sue preferenze o i suoi gusti cambino nel corso del tempo ma, semplicemente, il valore offerto dai brand alternativi si modifica, oppure, cambiando il contesto d'uso, è necessario cambiare anche il prodotto. Non solo, la teoria delle preferenze rivelate non spiega perché il consumatore scarti a priori alcune alternative scegliendo solo una o poche marche all'interno di una data categoria. Secondo la razionalità economica ipotizzata dall'economia tradizionale, il consumatore dovrebbe provare tutte le alternative per poi restare fedele a quella che incontra maggiormente le sue preferenze e i suoi gusti. Ma ciò non si verifica quasi mai. Il consumatore estromette semplicemente alcune alternative dal suo campo di scelta, senza peraltro disporre di tutte le informazioni che sarebbero necessarie per una decisione razionale!

Per far avanzare la comprensione del comportamento economico, occorre dunque decostruire i processi mentali che portano alle decisioni stesse. Si tratta di una nuova prospettiva, visto che è stato dimostrato che la decisione economica scaturisce dalla combinazione di processi controllati e automatici, emotivi e cognitivi ${ }^{7}$. Gran parte dei nostri acquisti non sono frutto di un'analisi delle informazioni disponibili e di deduzioni logiche, ma semplici reazioni viscerali a marcatori somatici; l'evoluzione della specie e la nostra esperienza hanno infatti lasciato segni indelebili nel nostro cervello emotivo e ci aiutano a scegliere rapidamente tra diverse opzioni. Tutte le volte che la nostra mente deve decidere se e cosa acquistare, siamo inconsciamente coinvolti in una tempesta di emozioni di diverso segno; è il prevalere delle emozioni di segno positivo sulle emozioni di segno negativo che porta alla decisione di acquisto.

7. Schneider W., Shiffrin R.M. (1977). Controlled and automatic human information processing: I. Detection, search and attention. Psycolog Rev 1: 1-66. Babiloni F., Meroni V.M., Soranzo R. Neuroeconomia, neuro marketing e processi decisionali. Springer (2007), pp. 23-24. Antonietti A , Balconi M. (2008). Mente ed economia. il Mulino. 


\section{G. Lugli}

Un mercato competitivo finisce per orientare necessariamente il comportamento delle imprese verso le preferenze dei consumatori scartando nel tempo i prodotti che non hanno ottenuto il favore del pubblico. Se si aspetta il verdetto ex post del consumatore, molte risorse vengono evidentemente sprecate. Basti pensare al numero di nuovi prodotti che vengono ritirati entro breve tempo dal lancio per non aver raggiunto il sell out previsto $^{8}$. Basti pensare ancora agli spot pubblicitari che non ottengono il risultato sperato di attenzione e memorizzazione. Questi errori e sprechi di risorse sono una naturale conseguenza del fatto che non è possibile scoprire ex ante cosa vuole il consumatore semplicemente chiedendoglielo, magari con la somministrazione di un questionario o un focus group. Spesso il consumatore non sa infatti cosa vuole e/o dà risposte cognitive diverse da quelle emotive che determineranno poi il suo comportamento di acquisto e consumo perché le risposte cognitive sono il frutto della mediazione culturale e dell'ambiente in cui avviene l'intervista; di conseguenza, si verifica di norma uno scostamento tra dichiarazioni e comportamento effettivo. Per superare questi ostacoli e migliorare la conoscenza del consumatore, sono state sviluppate metodologie che registrano l'impatto fisico di determinati stimoli. L'idea di fondo è che il corpo non può mentire e, di conseguenza, le misure biometriche possono arricchire gli strumenti di conoscenza del consumatore. Si tratta in particolare della misura dell'attività elettrica del cervello (EEG), della conduttanza cutanea, del battito cardiaco, della positron emitted tomography (PET), della magneto-encefalografia (MEG) e della functional magnetic resonance imaging (fRMI).

È appena il caso di ribadire che queste nuove tecniche di indagine non sostituiscono le vecchie, ma le completano. Infatti, una piena comprensione del comportamento umano richiede anche la conoscenza della costruzione individuale e sociale della realtà, che si può ottenere con le tecniche dell'intervista. La mediazione culturale, sociale e razionale, che determina le risposte dell'intervistato spiega infatti i comportamenti in cui prevale la componente cognitiva della mente. D'altra parte, la maggior affidabilità delle misure neuropsicologiche, che discende dall'insensibilità a fattori esterni e dalla minor possibilità di manipolazione intenzionale è comunque temperata da una ridotta validità ecologica. Le risposte ottenute in laboratorio non possono per definizione tener conto di tutte le variabili che determinano i comportamenti individuali e collettivi in ambienti reali.

8. Secondo l'IXP marketing group, 21.000 nuovi prodotti vengono inseriti ogni anno e solo il 10\% sopravvive ad un anno dal lancio (http://www.ixpg.com/brand-creation.html). 